\title{
Expression of the Parkinson's Disease-Associated Gene Alpha-Synuclein is Regulated by the Neuronal Cell Fate Determinant TRIM32
}

\author{
Maria Angeliki S. Pavlou ${ }^{1} \cdot$ Nicoló Colombo $^{2} \cdot$ Sandra Fuertes-Alvarez $^{3}$. \\ Sarah Nicklas $^{1}$ - Laura Gonzalez Cano ${ }^{1}$ - Maria C. Marín ${ }^{3}$ - Jorge Goncalves ${ }^{2}$. \\ Jens C. Schwamborn ${ }^{1}$
}

Received: 25 February 2016 / Accepted: 14 June 2016

(C) Springer Science+Business Media New York 2016

\begin{abstract}
Alpha-synuclein is an abundant neuronal protein which has been associated with physiological processes like synaptic function, neurogenesis, and neuronal differentiation but also with pathological neurodegeneration. Indeed, alphasynuclein (snca) is one of the major genes implicated in Parkinson's disease (PD). However, little is known about the regulation of alpha-synuclein expression. Unveiling the mechanisms that control its regulation is of high importance, as it will enable to further investigate and comprehend the physiological role of alpha-synuclein as well as its potential contribution in the aetiology of PD. Previously, we have shown that the protein TRIM32 regulates fate specification of neural stem cells. Here, we investigated the impact of TRIM32 on snca expression regulation in vitro and in vivo in neural stem cells and neurons. We demonstrated that TRIM32 is positively influencing snca expression in a neuronal cell line, while the absence of TRIM32 is causing deregulated levels of snca transcripts. Finally, we provided
\end{abstract}

Electronic supplementary material The online version of this article (doi:10.1007/s12035-016-9989-9) contains supplementary material, which is available to authorized users.

Jens C. Schwamborn

jens.schwamborn@uni.lu

1 Developmental and Cellular Biology Group, Luxembourg Centre for Systems Biomedicine (LCSB), University of Luxembourg, 6 avenue du Swing, 4367 Belvaux, Luxembourg

2 Systems control group, Luxembourg Centre for Systems Biomedicine (LCSB), University of Luxembourg, 6 avenue du Swing, 4367 Belvaux, Luxembourg

3 Instituto de Biomedicina (IBIOMED), Department of Molecular Biology, Universidad de León, Campus de Vegazana, León 24071, Spain evidence that TRIM32 binds to the promoter region of snca, suggesting a novel mechanism of its transcriptional regulation. On the one hand, the presented data link the PDassociated gene alpha-synuclein to the neuronal cell fate determinant TRIM32 and thereby support the concept that PD is a neurodevelopmental disorder. On the other hand, they imply that defects in olfactory bulb adult neurogenesis might contribute to early PD-associated non-motor symptoms like hyposmia.

Keywords Alpha-synuclein · TRIM32 · Transcriptional regulation $\cdot$ Neuronal differentiation

\section{Introduction}

Parkinson's disease (PD) is the second most prevalent neurodegenerative disorder, affecting $1-2 \%$ of the population over the age of 65 [1-3]. It is characterised by the loss of dopaminergic neurons mainly in the substantia nigra pars compacta, resulting in several motor deficits [4]. The formation of protein aggregates, known as Lewy bodies are considered as a major hallmark of the disease [5]. Braak et al. described in 2003 for the first time six stages that depict the progression of the disease. According to his classification, the first three stages are correlated with a pre-symptomatic phase of PD, while stages 3-6 are related to the symptomatic phase, (stage 3 is characterised with both pre-symptomatic and symptomatic phases). More precisely, according to his observations Lewy bodies first appear in the enteric and peripheral nervous system, and in non-dopaminergic structures of the lower brainstem and in the olfactory bulb $(\mathrm{OB})$, and then afterwards they attain the substantia nigra, midbrain, forebrain and neocortex [6]. 
Although the majority of the PD cases are idiopathic, some known genetic mutations are responsible for the onset of PD in around $10 \%$ of all cases [7, 8]. Eighteen genes are now implicated in the pathogenesis of PD, such as the autosomal dominant genes snca, lrrk2 or vps35, and the autosomal recessive genes pink1, parkin or $d j-1$ [9]. Patients with point mutations in the snca gene present a relatively early age of disease onset, with a mean age of 45.6 years [10]. Alphasynuclein (SNCA) is an abundant neuronal protein, mainly linked to synaptic function [5]. Several mutations and multiplications rendered snca to be classified as a PD-related autosomal dominant gene responsible for familial cases [9], while also being implicated in a wide range of diseases classified as alpha-synucleinopathies. SNCA pathology mainly contributes to the intracellular inclusion body formation, the Lewy bodies $[4,5]$. SNCA containing aggregates appear in the OB of PD patients at an early stage. In addition, the olfactory system is one of the first regions of the brain being affected in PD [11, 12] and it is also implicated in hyposmia and anosmia two of the non-motor symptoms of the disease [6]. Therefore, the snca gene seems to be an important candidate for the initiation and progression of PD.

Mice overexpressing snca, were shown to be greatly affected in certain brain regions, especially the $\mathrm{OB}$ and the dentate gyrus (DG). Excess of SNCA was shown to cause a delay in cell cycle entry of neural progenitor cells [13]. Furthermore, its overexpression is associated with increased cell death in the olfactory bulb (OB) and the DG [14-16]. In another study, neurogenesis was investigated in vivo and in vitro in snca transgenic mice (overexpressing snca) and in mouse embryonic stem cells (mESC) submitted to differentiation, respectively. For the latter, it was shown that neurogenesis was severely impaired as differentiated mESCs overexpressing snca presented a reduced expression of the neuronal marker $\beta$-III tubulin. In line with this, the snca transgenic mice exhibited a decrease in the number of proliferating cells and neuroblasts in the hippocampus, showing that this gene is linked with the generation of new neurons [14].

The OB comprises one of the two neurogenic regions in the adult mammalian brain where constantly, new neurons are generated. Adult OB neurogenesis is initiated in the subventricular zone (SVZ), from where immature neurons start migrating along the rostral migratory stream (RMS) towards the OB. After reaching the OB immature neuroblasts differentiate into inhibitory interneurons. Eventually, these new neurons are integrated into the existing local neuronal network [17]. This process was shown to be essential for olfaction [18] whereas deregulated adult neurogenesis has an impact on murine social interaction [19], on olfactory capabilities [20] and also contributes to Parkinson's diseaseassociated hyposmia [21].

Neurogenesis is regulated by the so-called cell fate determinants. The protein TRIM32, which belongs to the TRIM-
NHL family, functions as such a cell fate determinant. TRIM32 possess an E3 ubiquitin ligase activity. Among the ubiquitination targets are cell cycle regulators like p73 [22] and c-Myc [23]. Additionally, it was also shown that TRIM32 is able to associate with the RNA-induced silencing complex and the RNA helicase DDX6 in order to increase the activity of microRNAs [24]. Previously, we have shown that the cell fate determinant TRIM32 suppresses proliferation and is able to induce neuronal differentiation of neural stem cells (NSC) in embryonic [23, 25] and adult mouse brain [26]. In the absence of TRIM32, adult neuroblasts of the subventricular zone-olfactory bulb (SVZ-OB) system undergo extended proliferation and show delayed differentiation and less cell death, leading to an overproduction of adult generated neurons in the OB [26]. Finally, we have recently provided evidence that TRIM32 knock-out (ko) mice, present impaired olfactory capabilities as a result of increased neurogenesis and decreased apoptosis in the OB [20].

Since both TRIM32 and SNCA are involved in adult neurogenesis which is a key characteristic of the olfactory system, we set out to study a putative association between them. Since TRIM32 translocates to the nucleus during neuronal cell fate specification $[25,26]$, we hypothesised that TRIM32 transcriptionally regulates snca. So far, only few proteins have been identified to bind directly to the snca promoter and regulating its expression [27-29]. SNCA not only has a dominant role in PD pathology, but is also implicated in neurogenesis affecting cell proliferation and death, and the number of newly formed neurons. Obtaining a deeper comprehension of the role of snca in the regulation/deregulation of adult neurogenesis, will allow us to come to a closer understanding of the spectrum of the snca-associated diseases and pave the way towards potential novel therapeutic approaches. In summary, here, we describe TRIM32 as a novel regulator for the expression of the PD-associated gene snca. Furthermore, we present supporting information that TRIM32 is able to bind to the promoter of snca and that the absence of TRIM32 is responsible for abnormal snca messenger RNA (mRNA) levels both in vitro and in vivo.

\section{Results}

\section{TRIM32 Regulates Transcription of snca in a Cell Type-Specific Manner}

Since TRIM32 translocates into the nucleus upon differentiation ([30] and Online Resource 1) we speculated that TRIM32 may regulate the transcription of snca. In order to understand the existence of a possible relationship between snca and trim32, we designed a model based on previously published time series microarray data [31]. Time series measurements can unveil the causal structure of regulatory networks via the 
identification of associated dynamical models, in contrast to static measurements that usually only allow correlation and statistical (undirected) analysis of gene regulatory networks. Here, dynamical models are defined by differential equations describing the time evolution of certain observed input-output variables. To avoid overfitting, we chose the simplest model class: a first-order linear dynamical system. Hence, given the simplicity of the model, there is a very high confidence that links captured with this tool are in fact true links. The mathematical tools to obtain such systems from data are well described [32]. This methodology was applied to a mouse gene expression time series data set [31] to learn the regulatory network of the snca-trim 32 subsystem. The identified linear models (Fig. 1) reveal a clear regulatory effect of trim 32 on snca $(85 \%$ fit-where $100 \%$ is a perfect fit), but not vice versa ( $1 \%$ fit). Here, fitness is a measure of agreement between the simulated and real data [32]. In more details, to test whether trim 32 regulates snca, we looked for first-order linear dynamical systems that best fitted the data. We obtained $x_{\text {snca }}$ $=-0.2 x_{\text {snca }}+0.5 x_{\text {trim } 32}+e_{1}$ with fitness of $85 \%$ and where $x$ denotes the rate of change of $x$ over time and $e$ represents noise. The high fit indicates a strong evidence that trim 32 regulates snca. Repeating the analysis but with snca as the input and trim 32 as the output, the best model had a very poor fitness of $1 \%$, which indicates although trim 32 is likely regulating snca, the reverse case, that snca is regulating trim 32 , is not very likely.

In order to understand how specific this regulation of snca via trim 32 is, we used the same gene expression time series data to compare the regulatory effects of trim 32 on neuronal differentiation-associated genes (map2, neuroD, rbfox3) (Online Resource 2a) and potential housekeeping genes (chmpa2, gpi1, psmb2, rab7) (Online Resource 2c). Additionally, we tested whether the expression of the neuronal differentiation-associated genes and the housekeeping genes correlates with snca expression (Online Resource 2b, d). The result of this analysis suggests a non-negligible role of trim 32 on the regulation of other neuronal differentiation-associated genes, as well as the presence of other neuronal differentiation-associated genes involved in the regulation of snca. In summary, this analysis suggests that trim 32 might regulate the expression of snca, while it also regulates other genes involved in neuronal differentiation. Furthermore, expression of snca correlates well with the expression of neuronal differentiation-associated genes.

Based on these in silico data, we conducted luciferase assays using a vector encoding for the snca intron 1, which is considered to function as main promoter for $s n c a$, followed by a
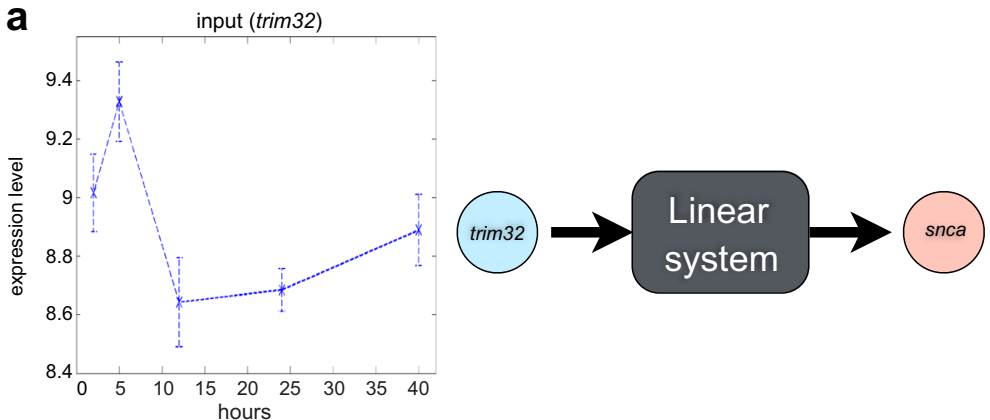

b
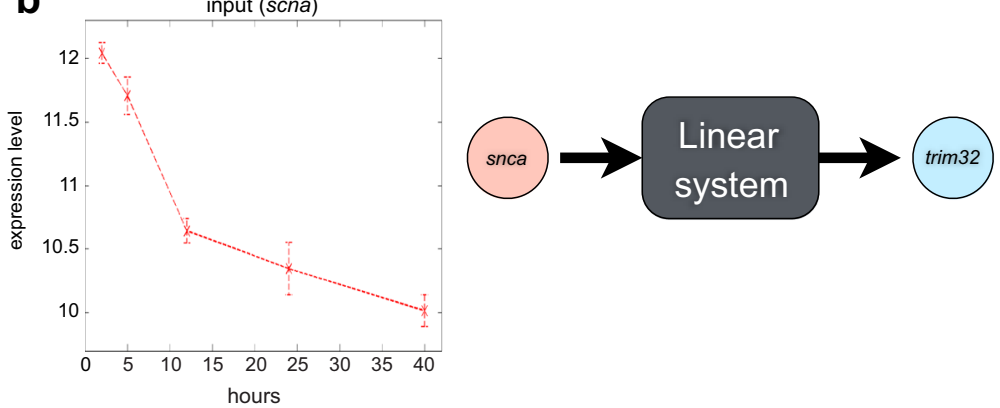
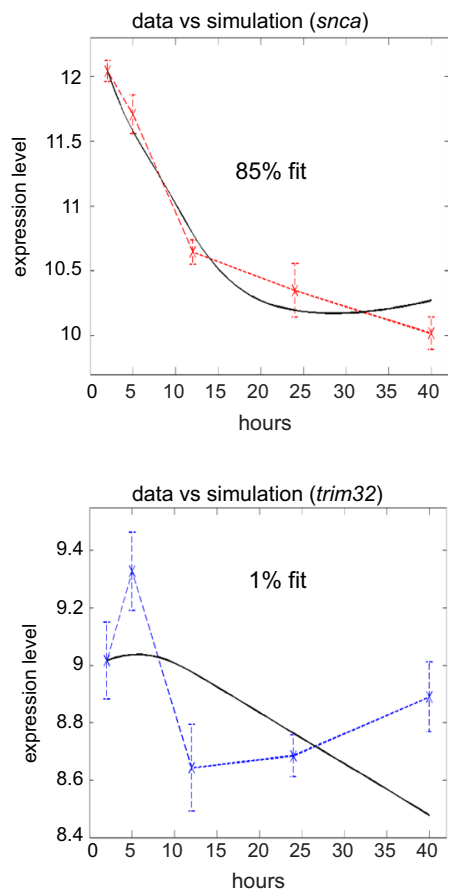

Fig. 1 Linear model depicting the dependency of snca on trim32. Diagram depicting the mRNA expression levels of snca (red) and trim32 (blue) during neuronal differentiation of root ganglion cells, which are plotted in relation to time, where each time point represents the mean and variance of the four replicates of the experimental data taken from Szpara et al. [31]. a The left depicts the input to the model (trim32). On the right is the output of the model (black), simulated with average initial conditions and driven by trim 32 , and the data from snca (red). The fitness of $85 \%$ can be verified by the good dynamical agreement of the data (snca) and the model driven by trim 32 . The simulation and high fitness empirically justify the simple model class assumption. b The reverse direction with snca driving trim 32 did not fit a first-order linear system, with the best fit being only $1 \%$ fit. The poor fit can be seen on the right side, which compares the simulation of the output of the model driven by snca with the data of trim 32 . The simulation also fails to capture the dynamical behaviour of trim 32 
the firefly luciferase gene (Fig. 2a). A basic vector containing solely the firefly luciferase was used as a control. Each construct was then transfected into neuroblastoma (N2a) cells together with different concentrations of TRIM32. The overexpression of TRIM32 led to a strong increase in the luciferase signal, demonstrating an increase in the transcriptional activity of the snca promoter (Fig. 2b).

When we repeated the same experiment in human embryonic kidney (HEK293T) cells, a low concentration of TRIM32 led to an increased luciferase activity compared to the control. On the other hand, when the concentration was increased, TRIM32 not only lost its ability to induce transcription of snca, but it even significantly decreased the transcriptional activity of the snca promoter (Online Resource 3). These data indicated that TRIM32 is able to alter the transcriptional activity of snca in a concentration and cell type-specific manner.

\section{TRIM32 is Able to Bind to the snca Promoter Sequence}

After showing that TRIM32 can activate the snca promoter in N2a cells, we next examined if TRIM32 binds to the snca promoter. To address this question, we performed chromatin immunoprecipitation (ChIP) assays in N2a cells, using a cocktail of anti-TRIM32 antibodies targeted against either the C- or $\mathrm{N}$-terminal domains of the protein. An IgG antibody was used as negative control and an anti-histone 3 (H3) antibody as positive control. We used several primers covering a $\sim 2.5 \mathrm{~kb}$ sequence upstream the transcription start site of the gene (Fig. 3a). Indeed, for all sequences investigated, we were able to detect a strong interaction of TRIM32 with the snca promoter region (Fig. $3 b$ and Online Resource 4).

To further quantify this interaction a set of five pairs of primers was used after ChIP, that cover a 400-bp region upstream of the snca transcriptional start site (Fig. 3c). This region was shown to be necessary and sufficient for transcription initiation [33]. With this approach we were able to detect an enrichment of DNA after precipitation of TRIM32, which was strongly above background but somewhat weaker than the interaction of histone $\mathrm{H} 3$ with the snca promoter (Fig. 3d, e and Online Resource 5).

To further validate the specificity of the interaction, we knocked down TRIM32 in N2a cells by transfecting them with two different shRNA sequences targeting TRIM32. A shRNA scrambled sequence was used as a control. Seventytwo hours after transfection, cells were subjected to ChIP assays and extracted chromatin was analysed via quantitative reverse transcription PCR (RT-qPCR) as described earlier. Cells transfected with the shRNA scrambled sequence showed a DNA enrichment after precipitation of TRIM32, which follows the same pattern as seen before in the untransfected cells (Online Resource 6a). However, when TRIM32 was knocked down, the enrichment of DNA was dramatically decreased, being almost identical with the

\section{a}

chromosome 4:

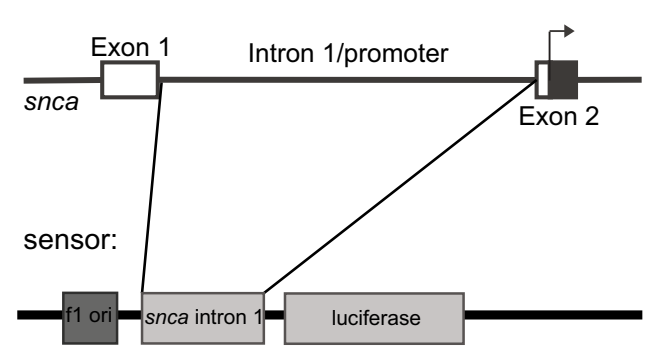

b

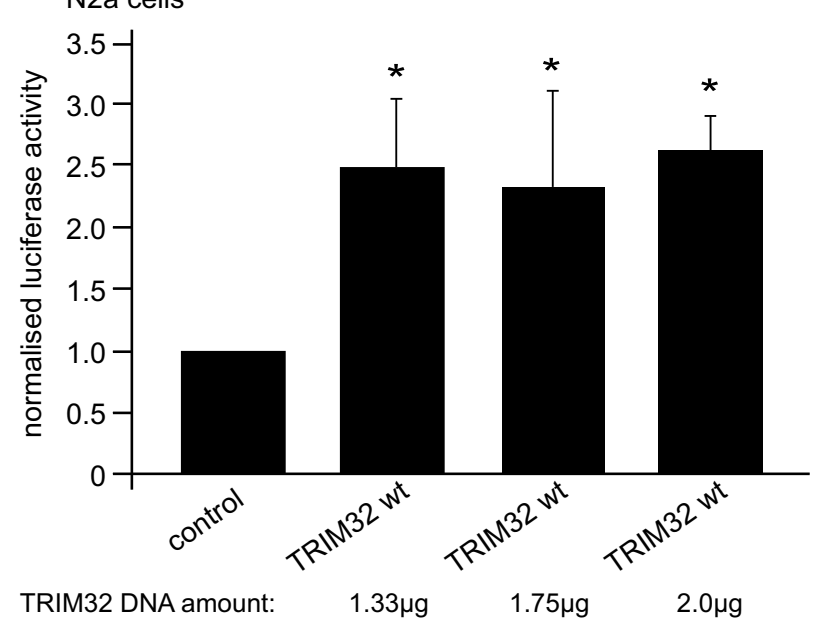

with $1.33 \mu \mathrm{g}$ of an empty vector and $1.33 \mu \mathrm{g}$ of the luciferase constructs (basic pGL3 vector or pGL3/snca intron 1) in N2a cells (mean $\pm \mathrm{SD} ; n=6$ independent experiments; Mann-Whitney $U$ test, $\left.{ }^{*} p \leq 0.05\right) ; 1.75 \mu \mathrm{g}$ of TRIM32 were coexpressed together with $0.75 \mu \mathrm{g}$ of an empty vector and $1.75 \mu \mathrm{g}$ of the luciferase constructs (basic pGL3 vector or pGL3/snca intron 1) in N2a cells (mean $\pm \mathrm{SD} ; n=6$ independent experiments; Mann-Whitney $U$ test, ${ }^{*} p \leq 0.05$ ); $2.0 \mu$ g of TRIM32 were coexpressed together with $2.0 \mu \mathrm{g}$ of the luciferase constructs (basic pGL3 vector or $\mathrm{pGL} 3 /$ snca intron 1 ) in $\mathrm{N} 2 \mathrm{a}$ cells (mean $\pm \mathrm{SD} ; n=3$ independent experiments; $t$ test, ${ }^{*} p \leq 0.05$ ) 
a
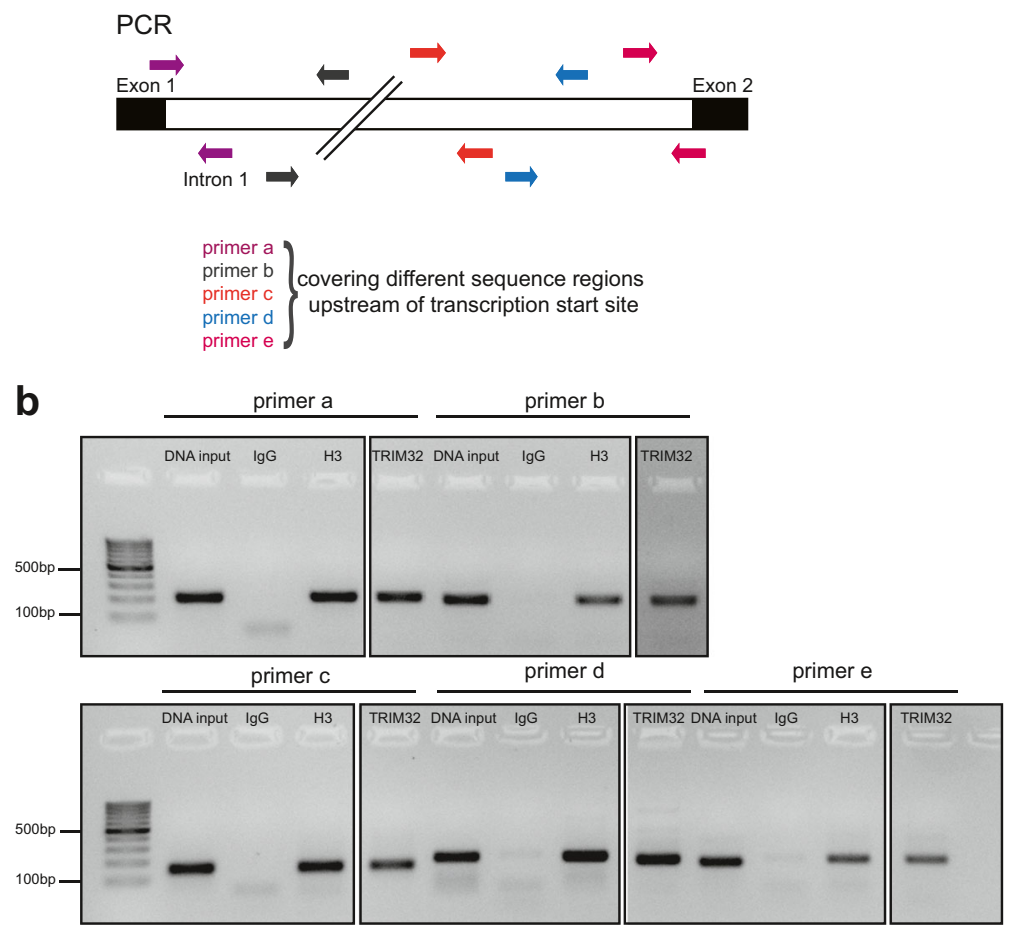

C

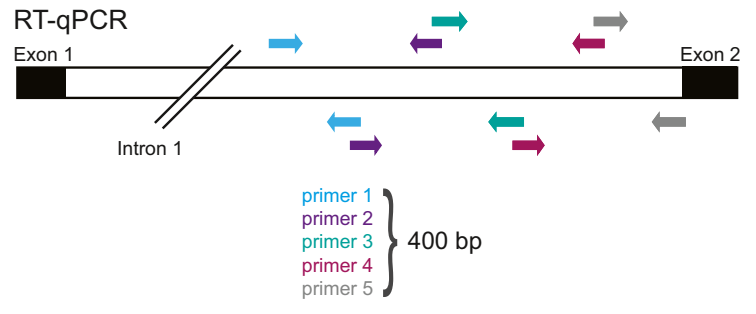

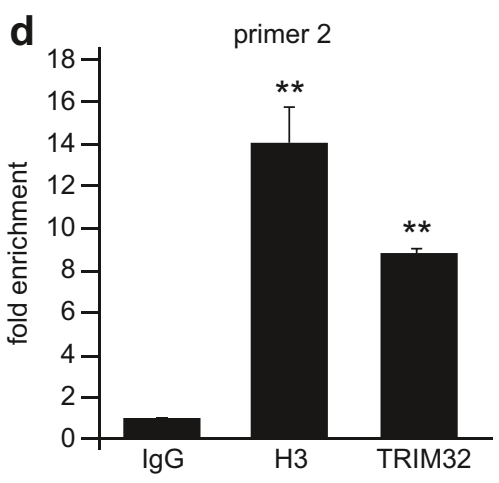

Fig. 3 TRIM32 interacts with the promoter region of snca. a Schematic representation of a fraction of snca gene, depicting the 5 representative out of the 16 primer pairs that were designed for PCR experiments, as an initial evaluation of the ChIP assays. b Agarose gel electrophoresis showing PCR results from ChIP-eluted DNA, with representative primer pairs of snca promoter region. An initial screening of the ChIP experiments was conducted by using PCR, with primers covering an approximate $2.5 \mathrm{~kb}$ genomic region. A 100-bp DNA ruler was used on the first lane of both upper and lower images and the samples are indicated. In total, 16 different primer pairs were designed in order to cover the above-mentioned large genomic region, but only five representatives were chosen to be shown. $\mathbf{c}$ Schematic representation of

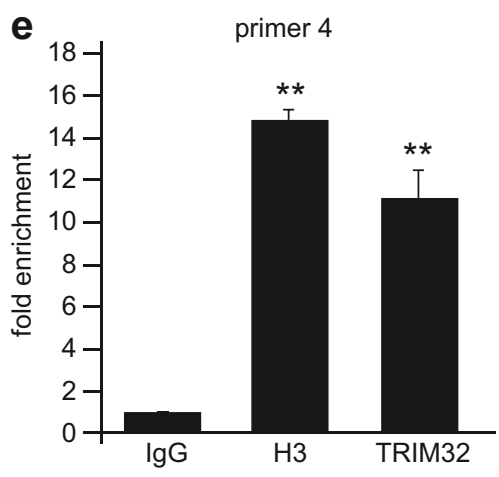

a fraction of snca gene, depicting the five primer pairs that were designed for RT-qPCR experiments. The five primer pairs are covering the last $400 \mathrm{bp}$ of intron 1, and were used to analyse the DNA retrieved from the immunoprecipitations. d, e RT-qPCR results showing the DNA fold enrichment under different immunoprecipitations. RT-qPCR was performed on the eluted DNA derived from chromatin immunoprecipitations either with the $\operatorname{IgG}$ (negative control), histone 3 (H3, positive control) or TRIM32 antibodies. Here, two out of five primer pairs are depicting the DNA fold enrichment when $\mathrm{H} 3$ or TRIM32 were used for the precipitation, in relation to IgG, meaning the background signal (mean $\pm \mathrm{SD} ; n=3$ independent experiments; $t$ test, $* p \leq 0.05, * * p \leq 0.001)$ 
negative control (Online Resource 6b). These results indicated that the observed interaction of TRIM32 with the snca promoter is specific and indeed, the observed interaction can be attributed to the existence of TRIM32. However, these data do not allow us to discriminate between the possibilities that TRIM32 either directly binds to the DNA or whether the interaction is mediated via additional chromatin-binding/associated factors.

\section{snca mRNA Expression Levels are Altered in TRIM32 ko and p73 ko mNSCs}

Because snca has been described to be upregulated during neuronal differentiation and maturation [34, 35], we investigated its mRNA expression levels in mouse NSCs (mNSCs) under stem cell maintenance conditions as well as after induction of differentiation.

To quantify the increase in snca expression during differentiation and to investigate whether this is regulated by TRIM32 we used wild type (wt) and TRIM32 ko mNSCs which were kept under maintenance conditions or subjected to neuronal differentiation for 5 days. In the ko mNSCs, indeed, the trim 32 transcript was absent as expected (Online Resource 7a). Furthermore, in wt mNSCs trim 32 was significantly upregulated during neuronal differentiation (Online Resource 7b). The snca mRNA expression levels were also upregulated in wt mNSCs subjected to neuronal differentiation (Fig. 4a). However, in TRIM32 ko mNSCs, this upregulation of snca upon neuronal differentiation was not detectable anymore (Fig. 4a). It is interesting to note that already, under maintenance conditions, TRIM32 ko mNSCs had slightly higher levels of snca compared to wt, which indicates that compensatory mechanisms, balancing the absence of TRIM32 may be active in these cells.

Since TRIM32 is a direct transcriptional target of TAp73 [22], we hypothesised that the impaired trim 32 expression observed in p73-deficient cells would have an impact on snca expression. To address this question, we additionally analysed trim 32 and snca mRNA levels in wt and p 73 ko mNSCs. The cells were similarly kept under maintenance conditions or were submitted to neuronal differentiation for 1,3 , or 5 days. trim 32 and snca levels were strongly upregulated during neuronal differentiation of wt mNSCs (Online Resource 7c and Fig. 4b, respectively). However, p73 ko mNSCs presented a blunted induction of trim32 (Online Resource 7c), which correlated with an almost complete impairment of snca induction during mNSC neuronal differentiation (Fig. 4b).

In addition, since we have shown that the TRIM32dependent regulation of snca might be cell type-dependent (Fig. 2b and Online Resource 3), we investigated the snca mRNA expression levels in wt and TRIM32 ko (Online Resource $8 \mathrm{a}, \mathrm{b}$ ) as well as in wt and p73 ko (Online

\section{a}
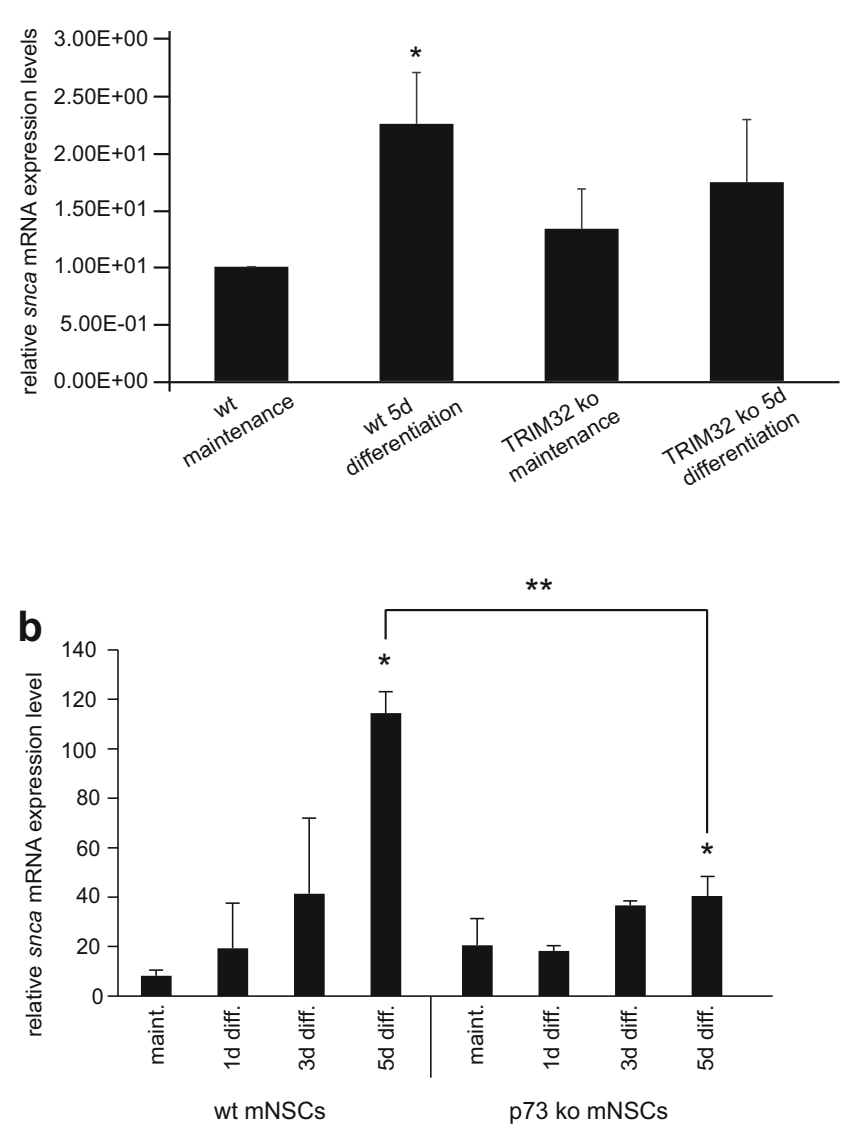

Fig. 4 mRNA levels of snca are deregulated during differentiation of TRIM32 ko and p73 ko mNSCs. a RT-qPCR measuring the relative snca mRNA expression levels in wt or TRIM32 ko mNSCs, under maintenance conditions and when cells are submitted for 5 days of neuronal differentiation. Note how mRNA levels were increased when wt mNSCs were subjected to neuronal differentiation, while TRIM32 ko mNSCs present no such significant increase. Values were normalised to GAPDH levels (mean $\pm \mathrm{SD} ; n=4$ independent experiments with $n=4$ different cell lines; Mann-Whitney $U$ test, ${ }^{*} p \leq 0.05$ ). b RT-qPCR measuring the relative snca mRNA expression levels in wt or p73 ko mNSCs, under maintenance conditions and when cells were submitted for 1,3 or 5 days of neuronal differentiation. Note how mRNA levels were increased in the mNSCs of both genotypes, though the rate of increase was significantly lower in the p 73 ko cells. Values were normalised to $18 \mathrm{~S}$ levels (mean $\pm \mathrm{SD} ; n \geq 3$ independent experiments; $t$ test, $\left.{ }^{*} p \leq 0.05,{ }^{* *} p \leq 0.001\right)$, maint maintenance

Resource 6c, d) mouse embryonic fibroblasts (MEFs). We were unable to detect any differences in the snca expression levels between the wt and TRIM32 ko MEFs (Online Resource $8 b$ ). These observations further strengthen the concept that the regulation of snca through TRIM32 is indeed cell type-specific. Furthermore, the provided data showing that the absence of only TRIM32 has an effect on the mRNA levels of snca during neuronal differentiation, might indicate the strong impact of TRIM32 on snca regulation and their importance in balanced neuronal differentiation. wt MEFs express low levels of snca, nevertheless, we detected a concomitant small, but significant, 
decrease in trim 32 and snca levels in p73-deficient cells (Online Resource 8c, d). Altogether, these data support our hypothesis that TRIM32 regulates the expression of snca.

\section{snca is Downregulated in the Olfactory Bulb of TRIM32 Deficient Mice}

The olfactory system is one of the first brain regions impaired in PD. Cells in the OB of PD patients show the typical presence of Lewy bodies which are mainly composed of alpha-synuclein aggregates [5, 11, 12]. Moreover, the fact that olfactory bulb (OB) neurogenesis is distorted when TRIM32 is absent [20], prompted us to investigate the mRNA levels of snca in different brain regions of adult wt and TRIM32 ko mice (Fig. 5a). As a further control for the specificity of our analysis approach, we additionally used a third mouse strain (C57BL/6JOlaHsd) which lacks expression of the snca gene due to a small chromosomal deletion [36]. RT-qPCR results confirmed that trim 32 expression is indeed absent in the TRIM32 ko mice and that snca is absent in C57BL/6JOlaHsd mice (Fig. 5b, c).

To investigate the potential differential expression of snca in the brain of TRIM $32 \mathrm{ko}$, mice tissue was dissected from the rostral migratory stream (RMS), the hippocampus (HP), the striatum, the cortex and the olfactory bulb. When the relative snca mRNA expression levels were investigated in these brain regions, only in the $\mathrm{OB}$ a significant difference was detectable (Fig. 5d). More precisely, the mRNA levels of snca were decreased in the OB of TRIM32 ko mice. These results highlight that the impaired neurogenesis observed in the absence of TRIM32 and the involvement of OB in PD might be interconnected through the regulation of snca expression via TRIM32.

a

b
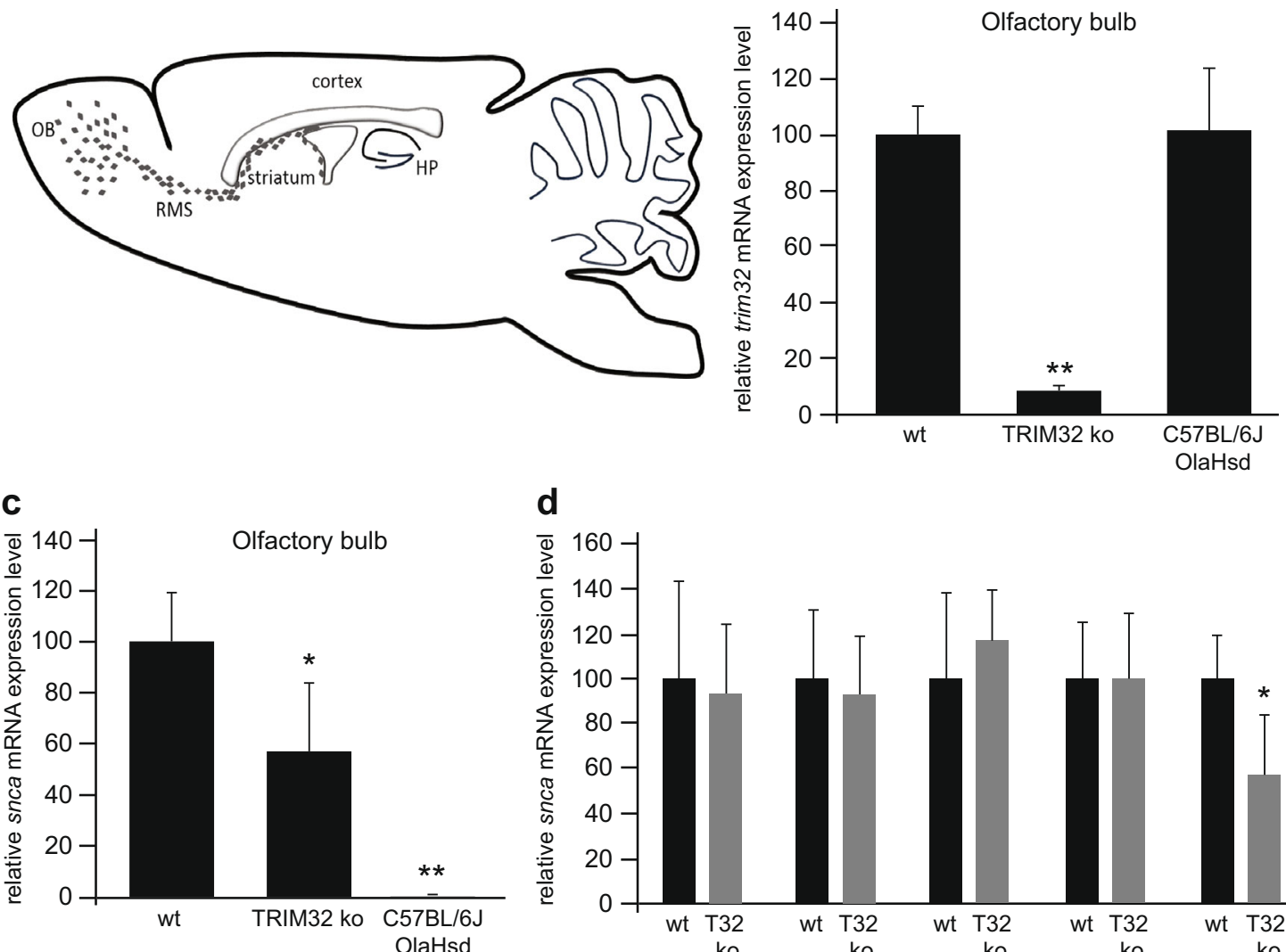

d

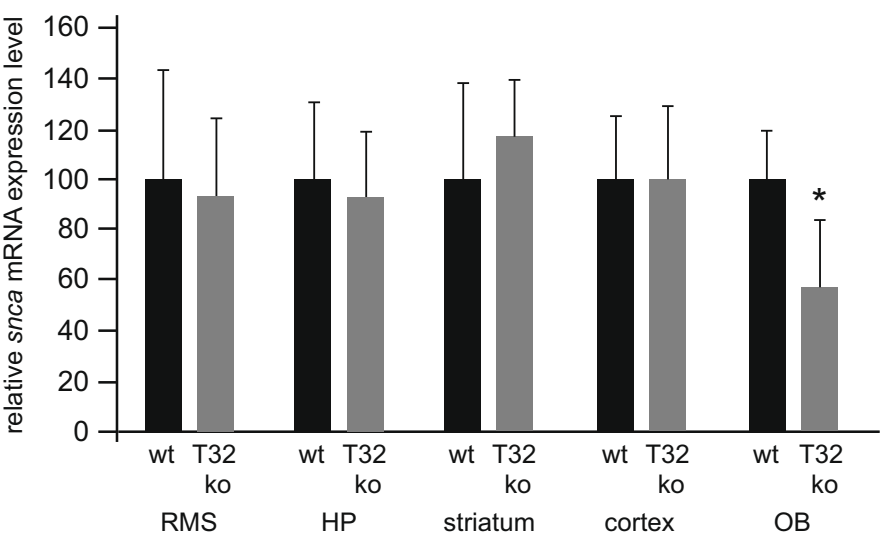

Fig. 5 mRNA levels of snca are decreased in the olfactory bulb of TRIM32 ko mice, but not in any other brain region. a Schematic representation of a sagittal mouse brain section, where the different brain regions that were dissected for the RNA isolation are shown. $\mathbf{b}$ RT-qPCR measuring the relative trim 32 mRNA expression levels in wt, TRIM32 ko and C57BL/6JOlaHsd mice' olfactory bulbs (OB, $\mathrm{N}_{\mathrm{wt}}=8$, $\mathrm{N}_{\text {TRIM32 ko }}=11, \mathrm{~N}_{\mathrm{C} 57 \mathrm{BL} / 6 \mathrm{JOlaHsd}}=6$ ). $\mathbf{c}$ RT-qPCR measuring the relative snca mRNA expression levels in wt, TRIM32 ko and C57BL/6JOlaHsd

mice' OB $\left(\mathrm{N}_{\mathrm{wt}}=8, \mathrm{~N}_{\text {TRIM32 ko }}=11, \mathrm{~N}_{\mathrm{C} 57 \mathrm{BL} / 6 \mathrm{JOlaHsd}}=6\right)$. d RT-qPCR measuring the relative snca mRNA expression levels in wt, TRIM32 ko and Ola mice' brain regions as indicated. Values were normalised to GAPDH levels, (Mean $\pm \mathrm{SD} ; \mathrm{N}_{\mathrm{wt}}=8, \mathrm{~N}_{\text {TRIM } 32 \text { ko }}=11$ for $\mathrm{OB}$ and $\mathrm{N}_{\mathrm{wt}}=4, \mathrm{~N}_{\text {TRIM32 ko }}=4$ for the other brain regions; $t$ test, $* p \leq 0.05, * *$ $C \leq 0.001)$. $H P$ hippocampus, $R M S$ rostral migratory stream, $O B$ olfactory bulb 


\section{Discussion}

In the present study, we provide evidence that the cell fate determinant TRIM32 is able to regulate the transcription of snca. Using luciferase assays, we obtained indications that overexpression of TRIM32 is positively affecting the transcription of snca. Interestingly, this effect was observed only when the neuronal N2a cell line was used. In non-neuronal HEK293T cells, low concentrations of TRIM32 induced activity of the snca promoter while high concentrations inhibited the activity. In addition, in the presented in silico data, a downregulation of snca via trim 32 was predicted though they are based on neuronal dorsal root ganglion cells. Altogether, these data indicate that TRIM32 regulates snca in a cell type, cell status and concentration-dependent manner. This conclusion is further supported by an analysis of snca mRNA expression in MEFs and mNSCs.

TRIM32 is associated with several diseases including limbgirdle muscular dystrophy type 2H [37], Bardet-Biedl syndrome [38], cancer [39, 40], autism spectrum disorders, attention deficit and hyperactivity disorder and epilepsy, and possibly other neurodevelopmental phenotypes [41-43]. Interestingly, in this context TRIM32 has been described to often have opposing roles in different cell types, e.g. it has been described to be pro- as well as anti-apoptotic [39, 40, 44-46] and to function as an oncogene as well as a tumour suppressor $[39,40,47]$. These opposing functions might be the consequence of cell type and concentration-dependent activities of TRIM32 that were described in this study as well.

TRIM32 has been described most intensively to function in neuronal cell fate specification during development and in the adult brain $[23,25,26]$. In particular, it regulates the generation of new neurons for the olfactory bulb [20]. Interestingly, problems with olfaction (hyposmia) are among the first nonmotor symptoms of PD [6] and aggregates of the PDassociated protein alpha-synuclein have been associated with this dysfunction [48, 49]. So far, there are only a few reports revealing proteins binding directly to the snca promoter or interacting with DNA response elements in order to form heterodimers and regulate transcription. Nurr1 [29], GATA-1 and GATA-2 [28] and ZSCAN21 and ZNF219 [27] have been identified to interact with the promoter region of snca and regulate its transcription. Here, we provided for the first time evidence that TRIM32 is interacting with the promoter region of this PD-related gene. Since TRIM32 has no known role of direct DNA binding, it remains to be shown whether it can interact with any of the documented proteins which are bound to the promoter region of the gene and how this putative interaction might additionally affect transcription. Our observation that the binding of TRIM32 is rather widespread within the investigated region of the snca promoter suggests that TRIM32 is probably not acting as a classical transcription factor. Instead of that TRIM32 might function as a structural protein or regulate the compaction of the chromatin. Finally, previous reports [50-53] have indicated a tight regulation of the snca intron 1 by methylation. Therefore, an involvement of TRIM32 in this process, which has an impact on gene expression, cannot be excluded.

Furthermore, as there is a solid connection between TRIM32 and neuronal defects [41, 42], our data further support this relationship. Additionally, a polymorphic microsatellite repeat region (NACP-Rep1), located $\sim 10 \mathrm{~kb}$ upstream of the transcription start site of the gene, was described to have a regulatory function on snca transcription [33, 54, 55]. The NACP-Rep1 repeat consists of a conserved repetition of nucleotides which acts as a negative modulator of snca expression, while the domains flanking the repeat have an inductive role [33]. Whether also this region is regulated by TRIM32 is currently unknown.

We have investigated the transcript levels of snca in wt and TRIM32 ko mNSCs under maintenance and neuronal differentiation conditions. wt mNSCs showed an upregulation of snca upon neuronal differentiation, which is consistent with other findings showing the upregulation of snca following differentiation $[35,56]$. When TRIM32 is missing, a deregulation is occurring in the snca mRNA levels of cells submitted to neuronal differentiation. These results were further supported by in vivo data, obtained from wt and TRIM32 ko mice where we compared the levels between different brain regions coming from adult tissue. Interestingly, these data, revealed significant differences between wt and ko mice in the mRNA levels of snca, only in the $\mathrm{OB}$ and not in tissue from any other brain region that was investigated (hippocampus, cortex, striatum and RMS). Already previously snca has been implicated with stem cell proliferation and maintenance [14-16, 57] and our results reinforce the idea that snca might indeed represent a crucial player in the balance of neural stem cell maintenance and differentiation during development. p73 has been described as a transcriptional regulator of trim 32 in neural progenitor cells [22]. mNSCs lacking p73 showed a decrease in the mRNA levels of both trim 32 and snca. One possible explanation would be that as a consequence of the decreased trim 32 mRNA levels in the p73 ko mNSCs, snca levels were also reduced. Interestingly, $\mathrm{p} 73$ regulates the expression of tyrosine hydroxylase, an enzyme that is critical for the synthesis of dopamine [58]. Therefore, $\mathrm{p} 73$ might be relevant for the development of PD at multiple levels.

Classically, PD is regarded as an age-associated neurodegenerative disorder. However, there is accumulating evidence that PD-associated genes play important roles during neuronal development [53]. As an example, it recently has been shown that alpha-synuclein regulates the amount of dopaminergic neurons that are specified during embryonic development in the substantia nigra [59]. These observations together with the here-described result that a PD-associated gene is controlled by a neuronal cell fate determinant make the hypothesis that 
PD actually is a neurodevelopmental disease very compelling. It is conceivable that PD-associated mutations in genes like snca, lrrk2, pink1, etc. affect their function during brain development and that as a consequence of this, the number, position or connectivity of dopaminergic neurons in the substantia nigra is affected. This defect might be compensated for a long time before it comes to the actually visible onset of the disease. Additionally, these developmental defects might not alone cause the disease but increase the susceptibility. Individuals with this developmentally caused increased susceptibility might react more sensitive to 'second hits' like toxins, neuroinflammation or protein aggregation.

In summary, our here-presented data suggest that the transcription of the snca gene is regulated by TRIM32. Since TRIM32 is highly expressed in the nucleus of cells of the $\mathrm{OB}$, this might represent a regulatory mechanism for the expression of snca in the OB. Consequently, TRIM32 appears to be an interesting new therapeutic target for modulation of PD. Additionally, these data imply that distorted olfactory bulb adult neurogenesis might contribute to early PD-associated non-motor symptoms like hyposmia. Finally, by linking the PD-associated gene alpha-synuclein to the neuronal cell fate determinant TRIM32, the concept that PD is a neurodevelopmental disorder is supported.

\section{Materials and Methods}

\section{Reagents and Plasmids}

For immunolabelling, the following antibodies were used: anti-Tuj1 (Covance), anti-NeuN (Millipore) and antiTRIM32-3149 (Gramsch Laboratories). For ChIP experiments, the following antibodies were used: anti-TRIM323150 (Gramsch Laboratories), anti-TRIM32-1137 (Gramsch Laboratories), anti-TRIM32-GS (Genescript) [25, 26, 60], anti-H3 pan (Diagenode) and IgG (Diagenode).

The following plasmids were used: pEGFP-N1 (Clontech Laboratories), pBABE-FLAG (Daniel Haber), MO93TRIM32/IRES-EGFP, two commercially available TRIM32 shRNA constructs (TRIM32 shRNA/IRES-GFP into pRNAT-H1.4/Retro vector, Genescript), a commercially available scrambled shRNA construct (shRNA scrambled/IRESGFP into pRNAT-H1.4/Retro vector, Genescript) and a commercially available snca siRNA (Dharmacon). The pGL3basic luciferase vector, the human intron 1 sequence of $\alpha$ synuclein subcloned into the pGL3-basic luciferase vector (LUC; Promega, Madison, WI, USA), the pIRES2-EGFP (Clontech) and human wt $\alpha$-synuclein subcloned into the pIRES2-EGFP were generously provided by Dr. Stefanis, Foundation for Biomedical Research of the Academy of Athens (IIBEAA), Athens, Greece.

\section{Mice}

TRIM32 - /- mice were obtained from the Mutant Mouse Regional Resource Centres (MMRRC) (https://www.mmrrc. org/catalog/sds.php?mmrrc_id=11810/011810.html) [26]. C57 $\mathrm{BL} / 6 \mathrm{JOlaHsd}$ mice were obtained from Harlan laboratories. Animals used in this study for breeding, maintenance and experimental procedures were treated in accordance with the German Federal law on the Care and Use of laboratory animals.

Animal experiments were conducted in agreement with European and Spanish regulations for the protection of experimental animals (Council Directive 86/609/CEE and RD$1201 / 2005$, respectively) with the appropriate institutional committee approval. Mice heterozygous for Trp73 on a mixed background C57BL/6 $\times 129 / \mathrm{svJae}$ [61] were backcrossed to C57BL/6, at least five times, to enrich for C57BL/6 background. Mice were genotyped as described earlier [61].

\section{Cell Culture}

Primary NSCs were isolated from single wild type (wt, TRIM32 +/+) and TRIM32 knock-out (TRIM32 ko) mouse brains at embryonic day (E) 12.5-14.5. NSCs were cultivated as neurospheres, as described before [24]. Neurospheres were kept under maintenance conditions in Dulbecco's modified Eagle's medium (DMEM)/Ham's F12 medium (PAA) supplemented with $10 \mathrm{ng} / \mathrm{mL}$ EGF (Peprotech), $10 \mathrm{ng} / \mathrm{mL}$ bFGF-2 (Peprotech), 1× N2 (Invitrogen), L-glutamine (PAA) and penicillin/streptomycin (PAA). Fifty percent exchange of the maintenance medium with DMEM/Ham's F12 medium (PAA) supplemented with $10 \mathrm{ng} / \mathrm{mL}$ bFGF-2 (Peprotech), $1 \times \mathrm{N} 2$ (Invitrogen), 1× B27 (Invitrogen), L-glutamine (PAA) and penicillin/streptomycin (PAA) was used to induce neuronal differentiation. NSCs were kept under differentiation for 5 days and then used for qPCR analysis or under differentiation for 5 days and then used for immunocytochemical stainings. For immunocytochemical staining, neurospheres were seeded onto coverslips coated with poly-ornithine (Sigma) and Laminin (Sigma).

Neuroblastoma (N2a) cells and human embryonic kidney (HEK293T, stably expressing the SV40 large T antigen) cells were cultivated on uncoated $10-\mathrm{cm}$ polystyrene tissue culture dishes in DMEM (Sigma) supplemented with $10 \%$ heatinactivated foetal calf serum (FCS) (PAA), L-glutamine (PAA) and penicillin/streptomycin (PAA). For transfection followed by luciferase assays, N2a cells and HEK293T cells were seeded onto poly-ornithine-coated 6-well polystyrene tissue culture plates 1 day prior to transfection. For transfection, followed by ChIP assays, N2a cells were seeded on non-coated $10-\mathrm{cm}$ polystyrene tissue culture plates 1 day prior to transfection.

wt and TRIM32 ko mouse embryonic fibroblasts (MEF) were prepared from 12-day-old wt and TRIM32 ko embryos as described elsewhere [62]. In total, three different pairs 
(from three different litters) were generated for the purposes of the experiments.

Independent embryonic cultures of wt and p73 ko mNSCs were initiated by dissecting the $\mathrm{OB}$ of at least three different E1.45 embryos and mechanically dissociating them as previously described [63]. The methods for primary culture of neural precursors cells by the neurosphere assay under mitotic conditions or under neuronal differentiation have been described before [64]. Briefly, differentiation was induced by seeding the $\mathrm{mNSCs}$ in differentiation medium (DMEM/Ham's F12, $0.6 \%$ glucose $4 \mathrm{mM}$ Hepes, $1 \%$ penicillin/streptomycin, $1 \%$ L-glutamine, $25 \mu \mathrm{g} / \mathrm{mL}$ insulin, $10 \%$ hormone mix, and $2 \%$ FBS). mNSCs were kept under differentiation for 1,3 , or 5 days and then used for qPCR analysis.

\section{Transfection}

N2a and HEK293T cells were transfected using Turbofect (Fermentas) according to manufacturer's instructions. Each transfection experiment was repeated at least three independent times.

\section{Luciferase Assays}

Luciferase signal was detected by using the Luciferase Assay System (Promega) according to the manufacturer's instructions. Briefly, N2a and HEK293T cells were transfected with $4 \mu \mathrm{g}$ of DNA per well, in 6well tissue culture plates. Each transfection was performed using either the pBABE-FLAG, the MO93TRIM32/IRES-EGFP or a combination of the above (pBABE-FLAG and MO93-TRIM32/IRES-EGFP), cotransfected with pGL3-basic luciferase vector or with pGL3-snca intron $1.72 \mathrm{~h}$ post-transfection, cells were lysed in passive lysis buffer (Promega). Luminescence measurements were performed in octuplicate in 96-well plates containing $20-\mu \mathrm{L}$ lysate per well. For each overexpression sample, the signal obtained from the pGL3snca intron 1 was normalised to that of the basic vector (pGL3).

\section{Immunocytochemistry}

For immunocytochemical staining, cells were fixed with $4 \%$ paraformaldehyde (PFA) in $120 \mathrm{mM}$ phosphate-buffered saline (PBS), pH 7.4 (4 \% PFA/PBS) followed by a $15 \mathrm{~min}$ permeabilisation using $0.05 \%$ Triton $\mathrm{X}-100$ in $\mathrm{PBS}$, at room temperature (RT). Cells were then blocked with $10 \%$ FCS in PBS for $1 \mathrm{~h}$ at RT and immunofluorescence staining was performed with primary and secondary antibodies diluted in blocking solution. For immunocytochemical staining of the mouse brains, the same protocol was used as described before [26].

\section{Microscopy}

Observation of TRIM32 localization on mouse sections and differentiated mNSCs was performed by acquiring images with a Zeiss confocal microscope. Image analysis was performed using ZEN lite (Zeiss), Adobe Photoshop and ImageJ software.

\section{Chromatin Immunoprecipitation}

Chromatin immunoprecipitation (ChIP) was performed using confluent N2a cells. The High Cell \# ChIP kit (Diagenode, Liege, Belgium) was used following the manufacturer's recommendations. Immunoprecipitations were conducted by using the mouse IgG (supplied with the kit), the anti-histone 3 antibody and a cocktail of anti-TRIM32 antibodies (TRIM32-3150, TRIM32-1137 and TRIM32-GS).

\section{PCR}

In order to primarily evaluate the eluted DNA which was obtained from the ChIP experiments, we performed PCR, followed by agarose gel electrophoresis. Sixteen different pairs of primers were used, covering a $\sim 2.5-\mathrm{kb}$ area, including exon1, intron 1 and a small region of exon 2 of the snca gene. The primers which were used for the experiments are listed below, (only representative images of a selection of primers are shown in the results):

snca-F1: 5'-AGCTGCTTGTCCCTTGTTTC-3', snca-R1: 5'-TGGGCACTGGAAGCTAAATC-3', snca-F2: 5' TTTAGCTTCCAGTGCCCAAC-3', snca-R2: 5' GATGCACAGGCTTTGAGACA-3', snca-F3: 5' TGTCTCAAAGCCTGTGCATC-3', snca-R3: 5' GGACCATCACAGGCAGTTTC-3', snca-F4: 5' CGGAAAGGTAAGAGGTGTGC-3', snca-R4: 5' CCATCTGTGGAGCAAGGAAT-3', snca-F5: 5' ATTCCTTGCTCCACAGATGG-3', snca-R5: 5' TTTTTACCCCGGAGGAGTCT-3', snca-F6: 5' CCTCCGGGGTAAAAACAAAT-3', snca-R6: 5' TTCCCTGCCTCAGTCTGTTC-3', snca-F7: 5' AACAGACTGAGGCAGGGAAG-3', snca-R7: 5' CTGTGGGTATCTGGAGCACA-3', snca-F 8: 5'CAGATACCCACAGCCCTCAC-3', snca-R 8: 5'GAACACCTGCTGGTCTCCTG-3', snca-F9: 5' CA GACGGCA GGA GACCAG, snca-R 9: 5' TTTGCTCCGGCTAGCTAAGA-3', snca-F10: 5' TAGCTAGCCGGAGCAAAGAG-3', snca-R10: 5' TGAAAAAGCAGATGCCACAG-3', snca-F11: 5' CTGCTTTTTCAAGCGGAAAC-3', snca-R11: 5' AAAGGCACCCTTCTTCCTCT-3', snca-F12: 5' GAGGAAGAAGGGTGCCTTTG-3', snca-R 12: 5' GCAGTCTCACTTGAGCACCA-3', snca-F13: 5'- 
TGGTGCTCAAGTGAGACTGC-3', snca-R 13： 5'AGAAGGAAGGGAGGGGAACT-3', snca-F14: 5'CCTCССТTCСТTCTCCAAAG-3', snca-R 14: 5'GCATCCTCTACCCCATCAAG-3', snca-F 15: 5'TGGGAACTTGATGGGGTAGA-3', snca-R15: 5' TGGGCACAGTGT TGATTGAT, snca-F16: $5^{\prime}$ CAACAATCAATCAACACTGTGC-3', snca-R16:5'GGCTAAAGATGTATTTTTGCT CCA-3'.

\section{RT-qPCR}

Mouse NSCs derived either from wt or TRIM32 ko mice were kept under maintenance conditions or were differentiated for 5 days. Total RNA was extracted by the RNeasy micro kit (QIAGEN, Venlo, Netherlands) following the manufacturer's recommendations.

For analysing the snca mRNA levels from different brain regions, adult wt, TRIM32 ko and C57BL/ 6JOlaHsd mice were used. Tissue from the rostral migratory stream (RMS), the cortex, the striatum, the hippocampus and the olfactory bulb (OB) was dissected under a binocular and was snap frozen. Total RNA was extracted by using the QIAzol lysis reagent (QIAGEN, Venlo, Netherlands) according to manufacturer's recommendations.

Complementary DNA (cDNA) was synthesised by using the High Capacity RNA-to-cDNA kit (Applied, Biosystems) according to manufacturer's recommendations. The cDNA synthesis was performed for $1 \mathrm{~h}$ at $37{ }^{\circ} \mathrm{C}$ and the reaction was stopped by heating to $95^{\circ} \mathrm{C}$ for $5 \mathrm{~min}$.

TRIM32 and snca expression levels were evaluated by the Maxima SYBR Green qPCR Master Mix (Thermo Fischer Scientific) following the manufacturer's recommendations. TRIM32 and snca levels were evaluated upon normalisation to glyceraldehyde-3-phosphate dehydrogenase (GAPDH) levels.

Total RNA from wt and p73 ko MEFs and mNSCs (passage 2) was extracted with TRI reagent (Ambion) and cDNA was prepared using SuperScript ${ }^{\mathrm{TM}}$ II First-Strand Synthesis System (Invitrogen) according to the manufacturer's instructions. The expression of cell cycle and apoptosis markers was detected by real-time quantitative RT-PCR in a StepOnePlus TM Real-Time PCR System (Applied Biosystems) using FastStart Universal SYBR Green Master (ROX) (Roche). Primers sequences and conditions were described before [22]. The expression levels of mRNA were expressed as $2^{\Delta \mathrm{Ct}}$ and normalised to $18 \mathrm{~S}$.

The primers which were used for the experiments are listed below:

TRIM32-F: 5'-GCATCCAGGAAGAGCTAG-3' TRIM32-R: 5'-CTCTACCACTTGACTGTTG-3' snca-F: 5'-GGTTCCAAAACTAAGGAAGG -3'
snca-R: 5'-CCTCCAACATTTGTCACTTG-3'

GAPDH-F: 5'-CTTTGGCATTGTGGAAGGGC-3'

GAPDH-R: 5'-TGCAGGGATGATGTTCTGGG-3'

Eluted DNA following the ChIP experiments was used for performing qPCR. Enrichment of the DNA was evaluated by the Maxima SYBR Green qPCR Master Mix (Thermo Fischer Scientific) following the manufacturer's recommendations. Results were analysed by using the fold enrichment method. Fold enrichment was calculated by using the formula $2^{-\mathrm{DDCt}}$, where DDCt is (Ct H3 or TRIM32) - ( Ct IgG). Used primers are listed below:

SNCA-F1: 5'- TAGCAGGCAAAGAGCTAGTGG-3'
SNCA-R1: 5'-CAGTCTTTGGAGAAGGAAGGG-3'
SNCA-F2: 5'-CCCTTCCTTCTCCAAAGAACTG-3'
SNCA-R2: 5'-TCCTCTACCCCATCAAGTTCC-3'
SNCA-F3: 5'-GGAACTTGATGGGGTAGAGGA-3'
SNCA-R3: 5'-TGTCACTTAAGGATGGGATGG-3'
SNCA-F4: 5'-CCATCCCATCCTTAAGTGACA-3'
SNCA-R4: 5'-AAAAATCAGTATGCCTTCCCC-3'
SNCA-F5: 5'-ATTTTTCCCCCTCAATTTCCT-3'
SNCA-R5: 5'-AAAGATGTATTTTTGCTCCACAC-3'

\section{Statistics}

Statistical analysis was performed by using the Student's $t$ test for single analyses. When normality tests failed, the MannWhitney rank sum test was used. $p$ values of $<0.05$ were considered significant.

\section{Regulatory Model from Microarray Data}

This section outlines how time series of microarray measurements have been extracted from the dataset of Szpara et al. [31] and used to fit linear models of the form:

$$
\begin{aligned}
& \dot{x}_{\text {snca }}=a_{1} x_{\text {snca }}+b_{1} x_{\text {trim } 32}+e_{1} \\
& \dot{x}_{\text {trim32 }}=a_{2} x_{\text {trim } 32}+b_{2} x_{\text {snca }}+e_{2}
\end{aligned}
$$

where $x$ represents the rate of change of $x$ over time, $a_{i}$ and $b_{i}$ are the parameters to be found and $e$ represents the noise and un-modelled dynamics. First, raw microarray data were downloaded from Szpara et al. [31] and processed using the $R$ functions rma( ) and exprs( ) in the oligo package [65] of http://bioconductor.org/biocLite.R. Expression data, for snca and trim 32 in all treatment and control replicates, have been extracted from the complete dataset and mapped back to natural scale from standard $\log _{2}$ scale. Two single expression time series data gene, $_{\text {gene }}=\{$ snca, $\operatorname{trim} 32\}$, were obtained by averaging the expression values at each time point, for $t=[2$, 5, 12, 24, 40] (in hours). Since the time points were not 
equally spaced, the time series have been interpolated using the MATLAB function $\hat{x}_{\text {gene }}=p \operatorname{chip}\left(t\right.$, data $\left.{ }_{\text {gene }}, t_{\text {new }}\right)$, where $t_{\text {new }}=[2,3,39]$. The parameters of the models were fitted with prediction error method MATLAB function, based on the algorithms in [32]. In particular, to estimate the transfer function we used the function tfest(Data,Options), where

Options $=\{$ 'InitialCondition' = 'estimate', 'InitMethod' $=$ 'n4sid' $\}$

and

Data $=$ iddata(Input,Output)

with Input $=\{$ trim 32, snca $\}$ and Output $=\{$ snca,, trim 32$\}$.

The fit was computed using compare (System,Output), where System is the identified model.

The study of the hypothesised regulatory role of trim 32 on snca has been completed by a time series analysis of (i) other possible regulatory roles of trim 32 and (ii) potential other regulators of snca. (i) The regulatory effects of TRIM32 on other genes, namely housekeeping genes [31] and neuronal differentiation-associated genes, have been investigated by repeating the previous analysis with trim 32 as an input and different genes as an output. In this approach the FIT between the single simulated input-output models and the data can be used to compare numerically the regulatory effect of trim 32 on the various housekeeping and neuronal differentiationassociated genes. (ii) To compare the effects of trim 32 on snca with the effect of other potential regulators of snca, the same analysis has been performed with different inputs (the same neuronal differentiation-associated genes and housekeeping genes) and snca as an output.

Acknowledgments The authors would like to thank Dr. Leonidas Stefanis and Dr. Daniel Haber for plasmids as well as Thea van Wuellen and Inga Werthschulte for excellent technical assistance.

\begin{abstract}
Author Contributions MASP did the conception and design, collection and assembly of data, data analysis and interpretation, manuscript writing and final approval of the manuscript. NC and JG did the model design and interpretation. SFA, LGC and MCM did the collection and assembly of data, data analysis and interpretation. SN did the generation of mNSCs lines. JCS did the conception and design, financial support, data analysis and interpretation, manuscript writing and final approval of the manuscript.
\end{abstract}

\section{Compliance with Ethical Standards}

Funding The J. C. S.'s lab is supported by the Boehringer Ingelheim Foundation and the fund "Innovative Medical Research" of the University of Münster Medical School, Schram-Stiftung (T287/21795/ 2011) by the Fonds National de la Recherche (FNR) Luxembourg (CORE, C13/BM/5791363), a University Luxembourg Internal Research Project (MidNSCs) and the EU Joint Programme Neurodegenerative Disease Research (JPND) project (supported by the FNR). L.G.C. was supported by a fellowship from the FNR (AFR, Aides à la Formation-Recherche). M.C.M 's lab is supported by Grant SAF2012-36143 from Spanish Ministerio de Ciencia e and LE310U14 from the Junta de Castilla y Leon. S.F.A holds a predoctoral contract (PIRTU) from Junta de Castilla y Leon.

\section{References}

1. de Rijk MC, Launer LJ, Berger K, Breteler MM, Dartigues JF, Baldereschi M, Fratiglioni L, Lobo A et al (2000) Prevalence of Parkinson's disease in Europe: a collaborative study of populationbased cohorts. Neurologic Diseases in the Elderly Research Group. Neurology 54(11 Suppl 5):S21-3

2. de Lau LM, Breteler MM (2006) Epidemiology of Parkinson's disease. Lancet Neurol 5(6):525-35. doi:10.1016/S14744422(06)70471-9

3. Van Den Eeden SK, Tanner CM, Bernstein AL, Fross RD, Leimpeter A, Bloch DA, Nelson LM (2003) Incidence of Parkinson's disease: variation by age, gender, and race/ethnicity. Am J Epidemiol 157(11):1015-22

4. Shulman JM, De Jager PL, Feany MB (2011) Parkinson's disease: genetics and pathogenesis. Annu Rev Pathol 6:193-222. doi:10.1146/annurev-pathol-011110-130242

5. Vekrellis K, Rideout HJ, Stefanis L (2004) Neurobiology of alphasynuclein. Mol Neurobiol 30(1):1-21. doi:10.1385/MN:30:1:001

6. Braak H, Del Tredici K, Rub U, de Vos RA, Jansen Steur EN, Braak E (2003) Staging of brain pathology related to sporadic Parkinson's disease. Neurobiol Aging 24(2):197-211

7. Gasser T (2009) Molecular pathogenesis of Parkinson disease: insights from genetic studies. Expert Rev Mol Med 11:e22. doi:10.1017/S1462399409001148

8. Nuytemans K, Theuns J, Cruts M, Van Broeckhoven C (2010) Genetic etiology of Parkinson disease associated with mutations in the SNCA, PARK2, PINK1, PARK7, and LRRK2 genes: a mutation update. Hum Mutat 31(7):763-80. doi:10.1002/humu.21277

9. Sundal C, Fujioka S, Uitti RJ, Wszolek ZK (2012) Autosomal dominant Parkinson's disease. Parkinsonism Relat Disord 18(Suppl 1):S7-10. doi:10.1016/S1353-8020(11)70005-0

10. Golbe LI, Di Iorio G, Sanges G, Lazzarini AM, La Sala S, Bonavita V, Duvoisin RC (1996) Clinical genetic analysis of Parkinson's disease in the Contursi kindred. Ann Neurol 40(5):767-75. doi:10.1002/ana.410400513

11. Pearce RK, Hawkes CH, Daniel SE (1995) The anterior olfactory nucleus in Parkinson's disease. Mov Disord 10(3):283-7. doi: $10.1002 / \mathrm{mds} .870100309$

12. Del Tredici K, Rub U, De Vos RA, Bohl JR, Braak H (2002) Where does parkinson disease pathology begin in the brain? J Neuropathol Exp Neurol 61(5):413-26

13. Tani M, Hayakawa H, Yasuda T, Nihira T, Hattori N, Mizuno Y, Mochizuki H (2010) Ectopic expression of alpha-synuclein affects the migration of neural stem cells in mouse subventricular zone. J Neurochem 115(4):854-63. doi:10.1111/j.14714159.2010.06727.x

14. Crews L, Mizuno H, Desplats P, Rockenstein E, Adame A, Patrick C, Winner B, Winkler J et al (2008) Alpha-synuclein alters Notch-1 expression and neurogenesis in mouse embryonic stem cells and in the hippocampus of transgenic mice. J Neurosci 28(16):4250-60. doi:10.1523/JNEUROSCI.0066-08.2008

15. Desplats P, Spencer B, Crews L, Pathel P, Morvinski-Friedmann D, Kosberg K, Roberts S, Patrick C et al (2012) Alpha-synuclein induces alterations in adult neurogenesis in Parkinson disease models via p53-mediated repression of Notch1. J Biol Chem 287(38): 31691-702. doi:10.1074/jbc.M112.354522

16. Winner B, Lie DC, Rockenstein E, Aigner R, Aigner L, Masliah E, Kuhn HG, Winkler J (2004) Human wild-type alpha-synuclein impairs neurogenesis. J Neuropathol Exp Neurol 63(11):1155-66

17. Alvarez-Buylla A, Garcia-Verdugo JM (2002) Neurogenesis in adult subventricular zone. J Neurosci 22(3):629-34

18. Lazarini F, Lledo PM (2011) Is adult neurogenesis essential for olfaction? Trends Neurosci 34(1):20-30. doi:10.1016/j. tins.2010.09.006 
19. Feierstein CE, Lazarini F, Wagner S, Gabellec MM, de Chaumont F, Olivo-Marin JC, Boussin FD, Lledo PM et al (2010) Disruption of adult neurogenesis in the olfactory bulb affects social interaction but not maternal behavior. Front Behav Neurosci 4:176. doi:10.3389/fnbeh.2010.00176

20. Hillje A-L, Beckmann E, Pavlou M, Jaeger C, Pacheco MP, Sauter T, Hiller K, Schwamborn JC, et al. (2015) The neural stem cell fate determinant TRIM32 regulates complex behavioral traits. Front Cell Neurosc 9. doi:10.3389/fncel.2015.00075

21. Marxreiter F, Regensburger M, Winkler J (2013) Adult neurogenesis in Parkinson's disease. Cell Mol Life Sci 70(3): 459-73. doi:10.1007/s00018-012-1062-x

22. Gonzalez-Cano L, Hillje AL, Fuertes-Alvarez S, Marques MM, Blanch A, Ian RW, Irwin MS, Schwamborn JC et al (2013) Regulatory feedback loop between TP73 and TRIM32. Cell Death Dis 4:e704. doi:10.1038/cddis.2013.224

23. Schwamborn JC, Berezikov E, Knoblich JA (2009) The TRIMNHL protein TRIM32 activates microRNAs and prevents selfrenewal in mouse neural progenitors. Cell 136(5):913-25. doi:10.1016/j.cell.2008.12.024

24. Nicklas S, Okawa S, Hillje AL, Gonzalez-Cano L, Del Sol A, Schwamborn JC (2015) The RNA helicase DDX6 regulates cellfate specification in neural stem cells via miRNAs. Nucleic Acids Res 43(5):2638-54. doi:10.1093/nar/gkv138

25. Hillje AL, Worlitzer MM, Palm T, Schwamborn JC (2011) Neural stem cells maintain their stemness through protein kinase C zetamediated inhibition of TRIM32. Stem Cells 29(9):1437-47. doi:10.1002/stem.687

26. Hillje AL, Pavlou MA, Beckmann E, Worlitzer MM, Bahnassawy L, Lewejohann L, Palm T, Schwamborn JC (2013) TRIM32dependent transcription in adult neural progenitor cells regulates neuronal differentiation. Cell Death Dis 4:e976. doi:10.1038 /cddis.2013.487

27. Clough RL, Dermentzaki G, Stefanis L (2009) Functional dissection of the alpha-synuclein promoter: transcriptional regulation by ZSCAN21 and ZNF219. J Neurochem 110(5):1479-90. doi:10.1111/j.1471-4159.2009.06250.x

28. Scherzer CR, Grass JA, Liao Z, Pepivani I, Zheng B, Eklund AC, Ney PA, Ng J et al (2008) GATA transcription factors directly regulate the Parkinson's disease-linked gene alpha-synuclein. Proc Natl Acad Sci U S A 105(31):10907-12. doi:10.1073 /pnas.0802437105

29. Yang YX, Latchman DS (2008) Nurr1 transcriptionally regulates the expression of alpha-synuclein. Neuroreport 19(8):867-71. doi:10.1097/WNR.0b013e3282ffda48

30. Hillje AL, Worlitzer MM, Palm T, Schwamborn JC (2011) Neural stem cells maintain their stemness through protein kinase $\mathrm{C}$ zetamediated inhibition of TRIM32. Stem Cells 29((9):1437-47. doi:10.1002/stem.687;10.1002/stem.687, Dayton, Ohio

31. Szpara ML, Vranizan K, Tai YC, Goodman CS, Speed TP, Ngai J (2007) Analysis of gene expression during neurite outgrowth and regeneration. BMC Neurosci 8:100. doi:10.1186/1471-2202-8-100

32. Ljung L (1998) System identification. In: Procházka A, Uhlír J, Rayner PWJ, Kingsbury NG (eds) Signal analysis and prediction. Applied and numerical harmonic analysis. Birkhäuser, Boston, pp 163-73. doi:10.1007/978-1-4612-1768-8_11

33. Chiba-Falek O, Nussbaum RL (2001) Effect of allelic variation at the NACP-Rep1 repeat upstream of the alpha-synuclein gene (SNCA) on transcription in a cell culture luciferase reporter system. Hum Mol Genet 10(26):3101-9

34. Hegde ML, Jagannatha Rao KS (2003) Challenges and complexities of alpha-synuclein toxicity: new postulates in unfolding the mystery associated with Parkinson's disease. Arch Biochem Biophys 418(2):169-78

35. Satoh JI, Kuroda Y (2001) Alpha-synuclein expression is upregulated in NTera2 cells during neuronal differentiation but unaffected by exposure to cytokines and neurotrophic factors. Parkinsonism Relat Disord 8(1):7-17

36. Specht CG, Schoepfer R (2001) Deletion of the alpha-synuclein locus in a subpopulation of $\mathrm{C} 57 \mathrm{BL} / 6 \mathrm{~J}$ inbred mice. BMC Neurosci 2:11

37. Frosk P, Weiler T, Nylen E, Sudha T, Greenberg CR, Morgan K, Fujiwara TM, Wrogemann K (2002) Limb-girdle muscular dystrophy type $2 \mathrm{H}$ associated with mutation in TRIM32, a putative E3ubiquitin-ligase gene. Am J Hum Genet 70(3):663-72. doi:10.1086 1339083

38. Chiang AP, Beck JS, Yen HJ, Tayeh MK, Scheetz TE, Swiderski RE, Nishimura DY, Braun TA et al (2006) Homozygosity mapping with SNP arrays identifies TRIM32, an E3 ubiquitin ligase, as a Bardet-Biedl syndrome gene (BBS11). Proc Natl Acad Sci U S A 103(16):6287-92. doi:10.1073/pnas.0600158103

39. Kano S, Miyajima N, Fukuda S, Hatakeyama S (2008) Tripartite motif protein 32 facilitates cell growth and migration via degradation of Abl-interactor 2. Cancer Res 68(14):5572-80. doi:10.1158 10008-5472.CAN-07-6231

40. Albor A, Kulesz-Martin M (2007) Novel initiation genes in squamous cell carcinomagenesis: a role for substrate-specific ubiquitylation in the control of cell survival. Mol Carcinog 46(8): 585-90. doi: $10.1002 / \mathrm{mc} .20344$

41. Lionel AC, Tammimies K, Vaags AK, Rosenfeld JA, Ahn JW, Merico D, Noor A, Runke CK et al (2014) Disruption of the ASTN2/TRIM32 locus at 9q33.1 is a risk factor in males for autism spectrum disorders, ADHD and other neurodevelopmental phenotypes. Hum Mol Genet 23(10):2752-68. doi:10.1093/hmg/ddt669

42. Lo-Castro A, Curatolo P (2014) Epilepsy associated with autism and attention deficit hyperactivity disorder: is there a genetic link? Brain Dev 36(3):185-93. doi:10.1016/j.braindev.2013.04.013

43. Ruan CS, Wang SF, Shen YJ, Guo Y, Yang CR, Zhou FH, Tan LT, Zhou L et al (2014) Deletion of TRIM32 protects mice from anxiety- and depression-like behaviors under mild stress. Eur J Neurosci 40(4):2680-90. doi:10.1111/ejn.12618

44. Horn EJ, Albor A, Liu Y, El-Hizawi S, Vanderbeek GE, Babcock M, Bowden GT, Hennings H et al (2004) RING protein Trim32 associated with skin carcinogenesis has anti-apoptotic and E3ubiquitin ligase properties. Carcinogenesis 25(2):157-67. doi:10.1093/carcin/bgh003

45. Albor A, El-Hizawi S, Horn EJ, Laederich M, Frosk P, Wrogemann K, Kulesz-Martin M (2006) The interaction of Piasy with Trim32, an E3-ubiquitin ligase mutated in limb-girdle muscular dystrophy type $2 \mathrm{H}$, promotes Piasy degradation and regulates UVB-induced keratinocyte apoptosis through NFkappaB. J Biol Chem 281(35): 25850-66. doi:10.1074/jbc.M601655200

46. Ryu YS, Lee Y, Lee KW, Hwang CY, Maeng JS, Kim JH, Seo YS, You KH et al (2011) TRIM32 protein sensitizes cells to tumor necrosis factor (TNFalpha)-induced apoptosis via its RING domain-dependent E3 ligase activity against X-linked inhibitor of apoptosis (XIAP). J Biol Chem 286(29):25729-38. doi:10.1074 /jbc.M111.241893

47. Liu J, Zhang C, Wang XL, Ly P, Belyi V, Xu-Monette ZY, Young $\mathrm{KH}, \mathrm{Hu} \mathrm{W}$ et al (2014) E3 ubiquitin ligase TRIM32 negatively regulates tumor suppressor $\mathrm{p} 53$ to promote tumorigenesis. Cell Death Differ 21(11):1792-804. doi:10.1038/cdd.2014.121

48. Zhang S, Xiao Q, Le W (2015) olfactory dysfunction and neurotransmitter disturbance in olfactory bulb of transgenic mice expressing human A53T mutant alpha-synuclein. PLoS One 10(3): e0119928. doi:10.1371/journal.pone.0119928

49. Mahlknecht P, Iranzo A, Hogl B, Frauscher B, Muller C, Santamaria J, Tolosa E et al (2015) Olfactory dysfunction predicts early transition to a Lewy body disease in idiopathic RBD. Neurology 84(7):654-8. doi:10.1212/WNL.0000000000001265

50. Jowaed A, Schmitt I, Kaut O, Wullner U (2010) Methylation regulates alpha-synuclein expression and is decreased in Parkinson's 
disease patients' brains. J Neurosci 30(18):6355-9. doi:10.1523 /JNEUROSCI.6119-09.2010

51. Matsumoto L, Takuma H, Tamaoka A, Kurisaki H, Date H, Tsuji S, Iwata A (2010) CpG demethylation enhances alpha-synuclein expression and affects the pathogenesis of Parkinson's disease. PLoS One 5:e15522-e15522. doi:10.1371/journal.pone.001552210.1371 /journal.pone.0015522

52. Pihlstrom L, Berge V, Rengmark A, Toft M (2015) Parkinson's disease correlates with promoter methylation in the alphasynuclein gene. Mov Disord 30(4):577-80. doi:10.1002 /mds.26073

53. Tan YY, Wu L, Zhao ZB, Wang Y, Xiao Q, Liu J, Wang G, Ma JF, Chen SD (2014) Methylation of alpha-synuclein and leucine-rich repeat kinase 2 in leukocyte DNA of Parkinson's disease patients. Parkinsonism Relat Disord 20:308-313. doi:10.1016/j. parkreldis.2013.12.002

54. Chiba-Falek O, Touchman JW, Nussbaum RL (2003) Functional analysis of intra-allelic variation at NACP-Rep1 in the alphasynuclein gene. Hum Genet 113(5):426-31. doi:10.1007/s00439003-1002-9

55. Cronin KD, Ge D, Manninger P, Linnertz C, Rossoshek A, Orrison BM, Bernard DJ, El-Agnaf OM et al (2009) Expansion of the Parkinson disease-associated SNCA-Rep1 allele upregulates human alpha-synuclein in transgenic mouse brain. Hum Mol Genet 18(17):3274-85. doi:10.1093/hmg/ddp265

56. Hasegawa T, Matsuzaki M, Takeda A, Kikuchi A, Akita H, Perry G, Smith MA, Itoyama Y (2004) Accelerated alpha-synuclein aggregation after differentiation of SH-SY5Y neuroblastoma cells. Brain Res 1013(1):51-9. doi:10.1016/j.brainres.2004.04.018

57. Le Grand JN, Gonzalez-Cano L, Pavlou MA, Schwamborn JC (2015) Neural stem cells in Parkinson's disease: a role for neurogenesis defects in onset and progression. Cell Mol Life Sci 72(4):773-97. doi:10.1007/s00018-014-1774-1
58. Grespi F, Melino G (2012) P73 and age-related diseases: is there any link with Parkinson disease? Aging (Albany NY) 4(12):923-31

59. Garcia-Reitboeck P, Anichtchik O, Dalley JW, Ninkina N, Tofaris GK, Buchman VL, Spillantini MG (2013) Endogenous alphasynuclein influences the number of dopaminergic neurons in mouse substantia nigra. Exp Neurol 248:541-5. doi:10.1016/j. expneurol.2013.07.015

60. Nicklas S, Otto A, Wu X, Miller P, Stelzer S, Wen Y, Kuang S, Wrogemann K et al (2012) TRIM32 regulates skeletal muscle stem cell differentiation and is necessary for normal adult muscle regeneration. PLoS One 7(1):e30445. doi:10.1371/journal.pone.0030445

61. Yang A, Walker N, Bronson R, Kaghad M, Oosterwegel M, Bonnin J, Vagner C, Bonnet H et al (2000) p73-deficient mice have neurological, pheromonal and inflammatory defects but lack spontaneous tumours. Nature 404(6773):99-103. doi:10.1038/35003607

62. Bahnassawy L, Perumal TM, Gonzalez-Cano L, Hillje AL, Taher L, Makalowski W, Suzuki Y, Fuellen G et al (2015) TRIM32 modulates pluripotency entry and exit by directly regulating Oct4 stability. Sci Rep 5:13456. doi:10.1038/srep13456

63. Vicario-Abejon C, Yusta-Boyo MJ, Fernandez-Moreno C, de Pablo F (2003) Locally born olfactory bulb stem cells proliferate in response to insulin-related factors and require endogenous insulinlike growth factor-I for differentiation into neurons and glia. $\mathrm{J}$ Neurosci 23(3):895-906

64. Gonzalez-Cano L, Herreros-Villanueva M, Fernandez-Alonso R, Ayuso-Sacido A, Meyer G, Garcia-Verdugo JM, Silva A, Marques MM et al (2010) p73 deficiency results in impaired self renewal and premature neuronal differentiation of mouse neural progenitors independently of p53. Cell Death Dis 1:e109. doi:10.1038/cddis. 2010.87

65. Irizarry RA, Bolstad BM, Collin F, Cope LM, Hobbs B, Speed TP (2003) Summaries of Affymetrix GeneChip probe level data. Nucleic Acids Res 31(4):e15 